\title{
FRUSTRATED TOTAL INTERNAL REFLECTION SPECTRA OF DIAZOQUINONE-NOVOLAC PHOTORESIST FILMS
}

\author{
S. D. Brinkevich, ${ }^{a} *$ D. I. Brinkevich, ${ }^{a}$ V. S. Prosolovich, \\ S. B. Lastovskii, ${ }^{\text {and A. N. Pyatlitskic }}$
}

UDC 539.216.2

Radiation-induced effects in thin films of diazoquinone-novolac photoresists on silicon irradiated with high-energy electrons ( $5 \mathrm{MeV}$ ) were investigated by frustrated total internal reflection (FTIR) IR Fourier spectroscopy. It was found that irradiation with electrons at a dose of more than $3 \cdot 10^{15} \mathrm{~cm}^{-2}$ leads to decrease of the integral absorption at wave numbers in the region of $3700-400 \mathrm{~cm}^{-1}$. The intensity of bands due to the $-O-H$-and particularly aliphatic $-C-H$ - bonds is increased most strongly during electron irradiation. At a dose of $\Phi=1 \cdot 10^{17} \mathrm{~cm}^{-2} \mathrm{the}^{\mathrm{intensity}}$ of the bands due to the methylene $\left(-\mathrm{CH}_{2}-\right)$ and methyl $\left(-\mathrm{CH}_{3}\right)$ groups is comparable with the noise level. The intensity of the band at $\sim 1600 \mathrm{~cm}^{-1}$, due to the vibrations of the aromatic ring, does not change at radiation doses in the range of $3 \cdot 10^{14}-1 \cdot 10^{17} \mathrm{~cm}^{-2}$. The obtained experimental data indicate strong cross-linking of the polymeric components during irradiation with electrons. It was found that the radiation-induced changes in the FTIR spectra depend on the type of photoresist (FP9120 and S1813 G2 SP15). The experimental relationships of change in the optical characteristics of thin films of photoresists due to electrons are explained in terms of the radiation chemistry of diazoquinone-novolac resins.

Keywords: frustrated total internal reflection spectrum, diazoquinone-novolac photoresist, irradiation, electron, silicon.

Introduction. Polymeric composites are widely used as resists in the formation of electronic instruments in submicron and nanolithography [1-3]. Diazoquinone-novolac resists (DQN) have a dominant position among photoresists [4-6]. They consist of a mixture of phenolformaldehyde resins and light-sensitive naphthoquinone diazide usually in a ratio of 5:1 [6]. Mixtures of the acetates of alcohols and glycols are usually employed as solvents. The DNQ photoresists include the FP9120 and S1813 G2 SP15 positive photoresists, which are analogs optimized for the $g$ line of the mercury lamp $(\lambda=435.8 \mathrm{~nm})$.

By IR Fourier spectroscopy with frustrated total internal reflection (FTIR) it is possible to obtain qualitative and quantitative information on the composition and structure of organic compounds and their mixtures in the solid state of aggregation [7]. We note that there have not been any previous investigations of electron-irradiated films of DQN photoresists on monocrystalline silicon.

The aim of the present work was to investigate the effect of electron irradiation on the FTIR spectra of the various types of positive diazoquinone-novolac resists and of FP9120 and S1813 G2 SP15 in particular.

Experimental. Films of the positive diazoquinone-novolac photoresists FP9120 and S1813 G2 SP15 1.8- $\mu$ m-thick were deposited on the surface of silicon by centrifuging [8]. Plates of KEF-4,5 monocrystalline silicon $100 \mathrm{~mm}$ in diameter with (100) orientation were used as substrates. Before the film of photoresist was formed the silicon plates were submitted to a standard cycle of cleaning in organic and inorganic solvents. The thickness of the films of photoresist was monitored by a MII-4 microinterferometer at five fixed points located on two mutually perpendicular diameters on each plate. The films were irradiated by electrons with energy of $5 \mathrm{MeV}$ on a U-003 linear electron accelerator at doses of $3 \cdot 10^{14}-1 \cdot 10^{17} \mathrm{~cm}^{-2}$. The density of the electron beam $1 \cdot 10^{12} \mathrm{~cm}^{-2} \cdot \mathrm{s}^{-1}$ was monitored by means of a Faraday cylinder. The temperature of the samples in the process was not higher than $310 \mathrm{~K}$.

\footnotetext{
${ }^{*}$ To whom correspondence should be addressed.
}

${ }^{\mathrm{a} B e l a r u s i a n}$ State University, Minsk, 220030, Belarus; email: brinkevichsd@bsu.by; ${ }^{\mathrm{b}}$ Scientific-Practical Materials Research Centre, National Academy of Sciences of Belarus, Minsk, Belarus; email: lastov@ifttp.bas-net.by; ${ }^{\mathrm{c} J S C ~ I N T E G R A L, ~}$ Holding Management Company, Minsk, Belarus; email: petan@tut.by. Translated from Zhurnal Prikladnoi Spektroskopii, Vol. 87, No. 6, pp. 941-948, November-December, 2020. Original article submitted August 18, 2020. 